\title{
A SECURITY PERIOD UPDATE METHOD USING EVALUATION FUNCTION FOR IMPROVING ENERGY EFFICIENCY OF STATISTICAL EN-ROUTE FILTERING- BASED WSNS
}

\author{
Jung-Sub Ahn ${ }^{1}$ and Tae-Ho Cho ${ }^{2}$ \\ ${ }^{1}$ College of Information and Communication Engineering, \\ Sungkyunkwan University, Suwon 16419, Republic of Korea \\ ${ }^{2}$ College of Software Platform, Sungkyunkwan University, \\ Suwon 16419, Republic of Korea
}

\begin{abstract}
In recent years, Wireless Sensor Networks(WSNs) research has been carried out with the goals of achieving high security and energy efficiency. In a WSN, sensor nodes are vulnerable to physical attacks because they are deployed in an open environment. An attacker can inject a false report into networks using these vulnerabilities. F. Ye et al. proposed statistical en-route filtering to prevent false report injection attacks. In order to effectively use their scheme, techniques for determining thresholds using fuzzy logic have been studied. To effectively apply these techniques to the network, an appropriate update period should be set according to the network environments. In this paper, we propose a security period update method in order to improve the lifetime of the network in the statistical en-route filtering approach based on a wireless sensor network of the cluster environment. The experimental results show that up to an $11.96 \%$ improvement of the energy efficiency can be achieved when the security threshold is set to the optimal period.
\end{abstract}

\section{KEYWORDS}

Network Simulation, Wireless Sensor Network, Statistical En-route Filtering, False Report Injection Attack, Energy Efficiency.

\section{INTRODUCTION}

A wireless sensor network (WSN) consists of hundreds to thousands of sensor nodes and a base station (BS), providing real-time monitoring of sensor fields in industrial, medical, and military applications. A sensor node consists of a processor, memory, a battery, and a wireless transmitter [1-2]. Due to battery limitations, research involving increasing the network lifetime considering limit factors is currently actively studied [3]. If an event occurs, the sensor node generates a report with detected information and sends it to the BS using a hop-by-hop technique to notify the 
user. Sensor nodes are vulnerable to physical attacks because they have limited memory and batteries, and are deployed in open environments [4]. The attacker can compromise the sensor node and generate a false report using the secret information contained in the node. In addition, the attacker can inject a false report with the wrong event data type into the networks, as shown in Figure 1.

If a WSN consists of a cluster, false report insertion attacks are divided into two cases when the cluster head $(\mathrm{CH})$ node that generates the report is compromised and when the member $(\mathrm{MB})$ node that creates the authentication key is compromised. If the $\mathrm{CH}$ node is compromised, the attacker generates an arbitrary report and transmits it to the next node, causing false notification and energy exhaustion problems of intermediate nodes in the routing path [5-6]. If the MB node is compromised, it can generate a false event notification to the $\mathrm{CH}$ node, depleting the energy of the cluster region and deploy incapacitating nodes. To minimize this problem, it is necessary to detect and remove the false report early and to filter out incorrect alarms to users. To prevent false report injection attack, F. Ye et al. proposed statistical en-route filtering (SEF) [7]. In SEF, it is important to set an appropriate security threshold because the security threshold has a trade-off relationship between power consumption and filtering probability. Security threshold determining methods using fuzzy logic were proposed to obtain an appropriate security threshold value [8]. However, this method does not consider the update cycle of the fuzzy logic process. If the update period is not taken into consideration, the worst case consumes more power.

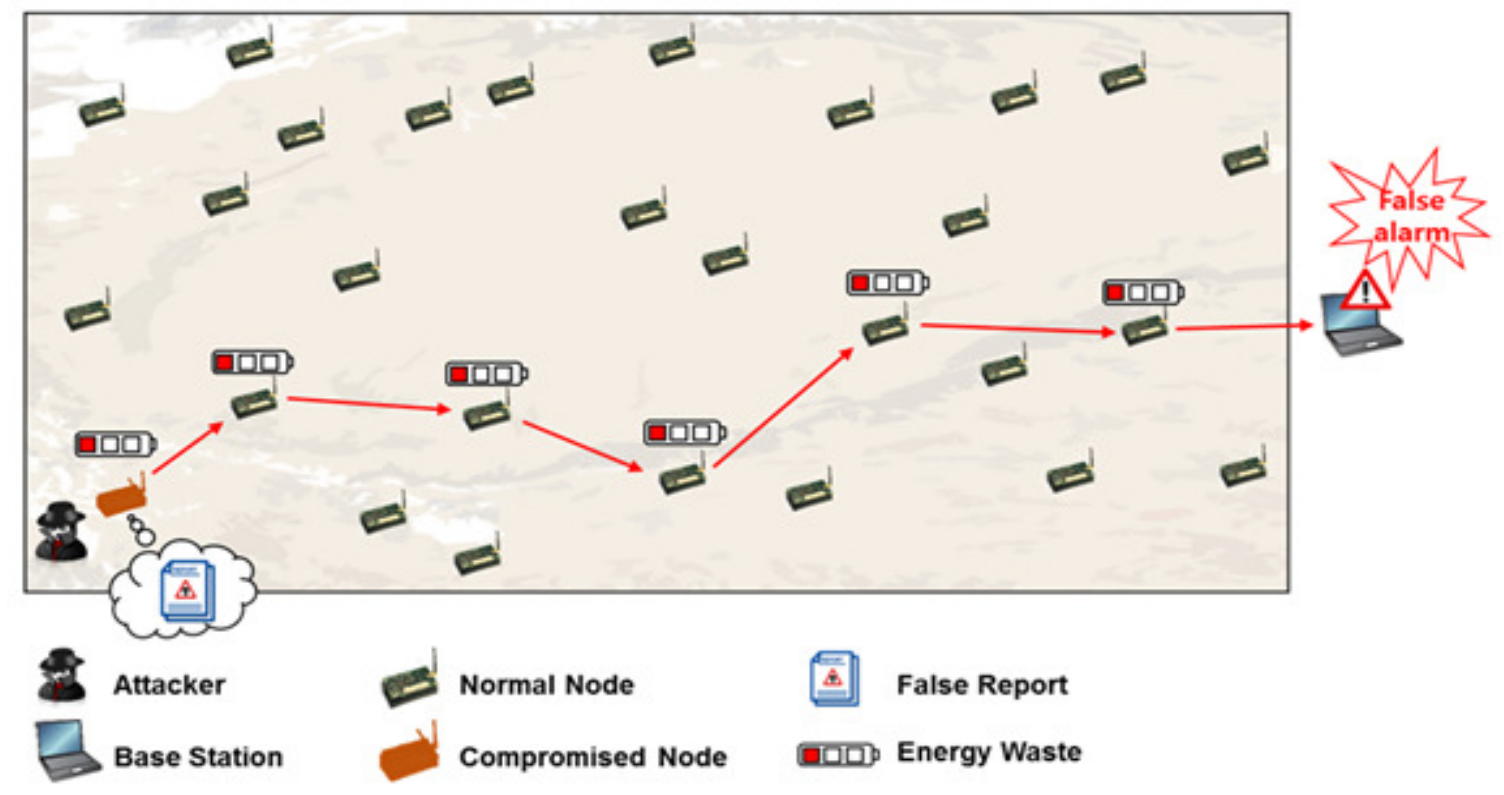

Figure 1. False report injection attack

In this paper, we propose a security period update method using an evaluation function to improve the energy efficiency of SEF-based WSNs. The proposed method automatically determines the update cycle considering network environment factors. Nodes that are updated on a periodic basis do not have to send the information messages needed for the update and can save energy by adjusting the security strength in a timely manner. We demonstrated the performance of the proposed method through performance analysis by applying the evaluation function. The experimental results show that the proposed method saves up to $11.96 \%$ of energy. 
The remainder of the paper is organized as follows. In Section 2, we explain the statistical enroute filtering scheme and motivation. Section 3 introduces the proposed evaluation function and fuzzy logic. Section 4 details the experiment results and, finally, the conclusions of this study are discussed in Section 5.

\section{RELATED WORKS}

\subsection{Statistical En-route Filtering (SEF)}

F. Ye et al. proposed SEF to prevent false report injection attacks. SEF statistically filters false reports by adding threshold values for authentication to the report generated by the representative node. The intermediate node verifies the report when a false report is transmitted. In addition, the intermediate nodes block false reports, thereby reducing unnecessary energy consumption to the BS. The SEF method consists of four phase: the key distribution phase, report generation phase, intermediate filtering phase, and BS node verification phase. In the key distribution phase, the user sets various setting values including the threshold value before the sensor nodes are deployed in the target area. The higher the threshold, the greater the false report detection rate, which makes it difficult for an attacker to generate false reports. However, high thresholds require high power consumption to transmit reports. Each node is randomly distributed among the key sets divided by the partition in the global key pool created at the BS. Figure 2 shows the key distribution process, where $\mathrm{P} 1$ to $\mathrm{pl}$ denote the partition containing the key.

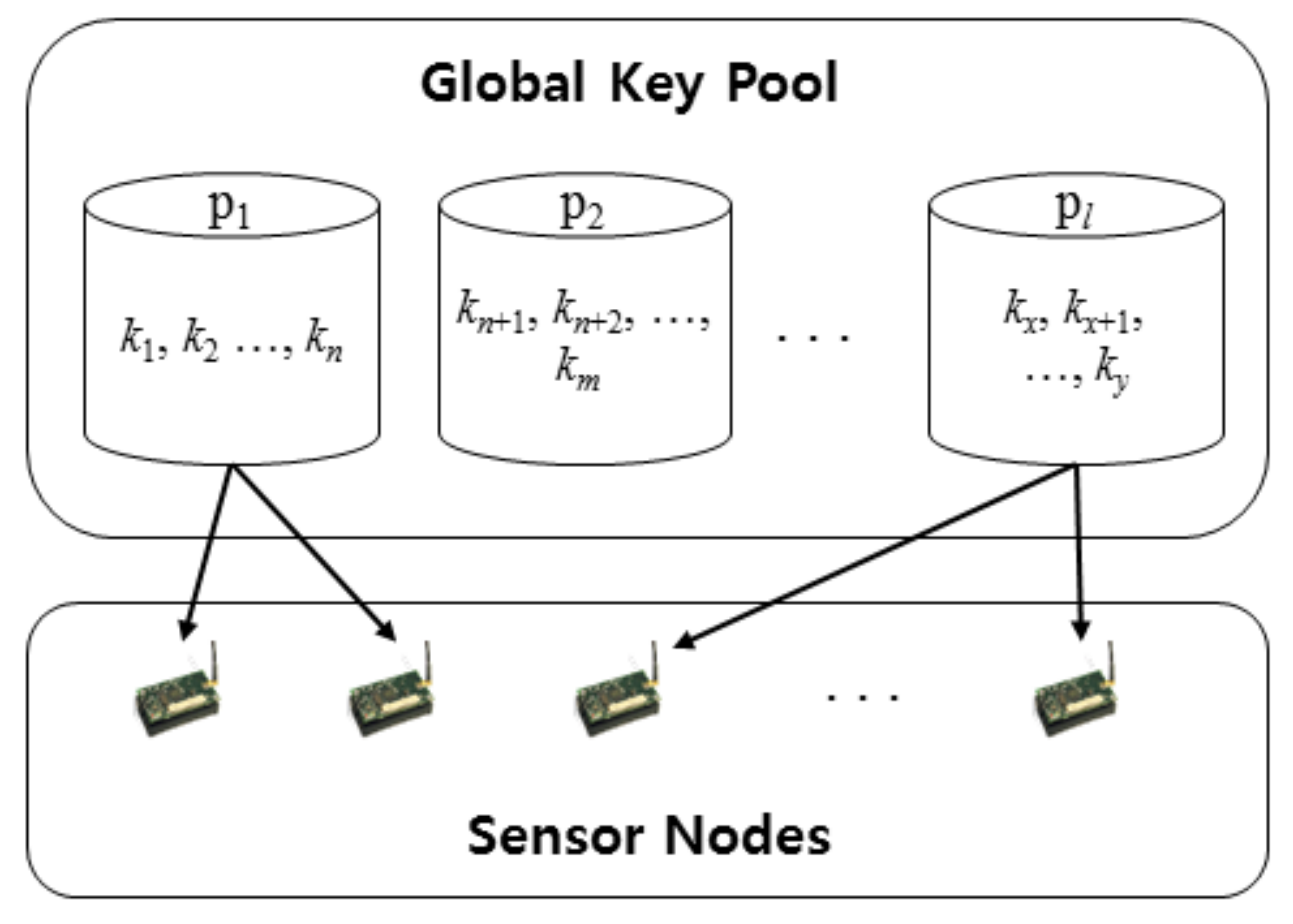

Figure 2. Key distribution phase in SEF

After the key distribution process is over, the nodes are deployed in the target area where they want to collect information. When the deployed sensor nodes detect the event, the node with the highest detection rate is selected as the representative node. The representative node broadcasts 
event information to find neighboring nodes that have detected the same event signal value. Neighboring nodes that have received the event information compare whether occurrence same event information. If the generated events are the same, the message authentication code (MAC) is generated and transmitted to the representative node using the pre-distributed key and hash function. The generated MACs are used to verify the report. The threshold value signifies the number of MACs included in the report when the representative node generates the report. If a MAC is collected that is smaller than the threshold for collecting MACs, no reports are generated. The representative node generates the report by including event contents and MACs that vary from one another as the threshold value. Since each node has a certain probability of a common key, it can probabilistically detect false reports. Event reports are transmitted to the BS node through multi-hop routing. If the forwarding node receives the report, it goes through the verification process shown in Figure 3

1) Check that $T\left\{\mathrm{i}_{\mathrm{j}}, \mathrm{M}_{\mathrm{ij}}\right\}$ tuples exist in the packet; drop the packet otherwise.

2) Check that the $T$ key indices $\left\{i_{j}, 1=j=T\right\}$ belong to $T$ instinct partitions; drop the packet otherwise.

3) If it has one key $\mathrm{K} \in\left\{\mathrm{K}_{\mathrm{ij}}, 1=\mathrm{j}=\mathrm{T}\right\}$, it computes $\mathrm{M}=\mathrm{MAC}\left(\mathrm{K}, L_{\mathrm{E}}|t| E\right)$ using Equation 1. Determine

if the corre sponding $\mathrm{M}_{\mathrm{j}}$ is the same as $\mathrm{M}$ If so, it sends the packet to the next hop; otherwise, the packet is dropped.

4) If it does not have any of the keys in $\left\{\mathrm{K}_{\mathrm{ij},}, 1=\mathrm{j}=\mathrm{T}\right\}$, send the packet to the next hop.

Figure 3. Four operation phases of en-route filtering

$\mathrm{M}_{\mathrm{ij}}$ refers to the MACs included in the report. The MAC consists of $\mathrm{K}$, which is the key value of the node, Le is the event information, $t$ is the event occurrence time, and $\mathrm{E}$ is the contents of the event. Finally, when the BS node receives the report, it verifies all MACs included in the report using the global key pool. If the BS node determines that it is a normal report after verification, it sends the event contents to the user.

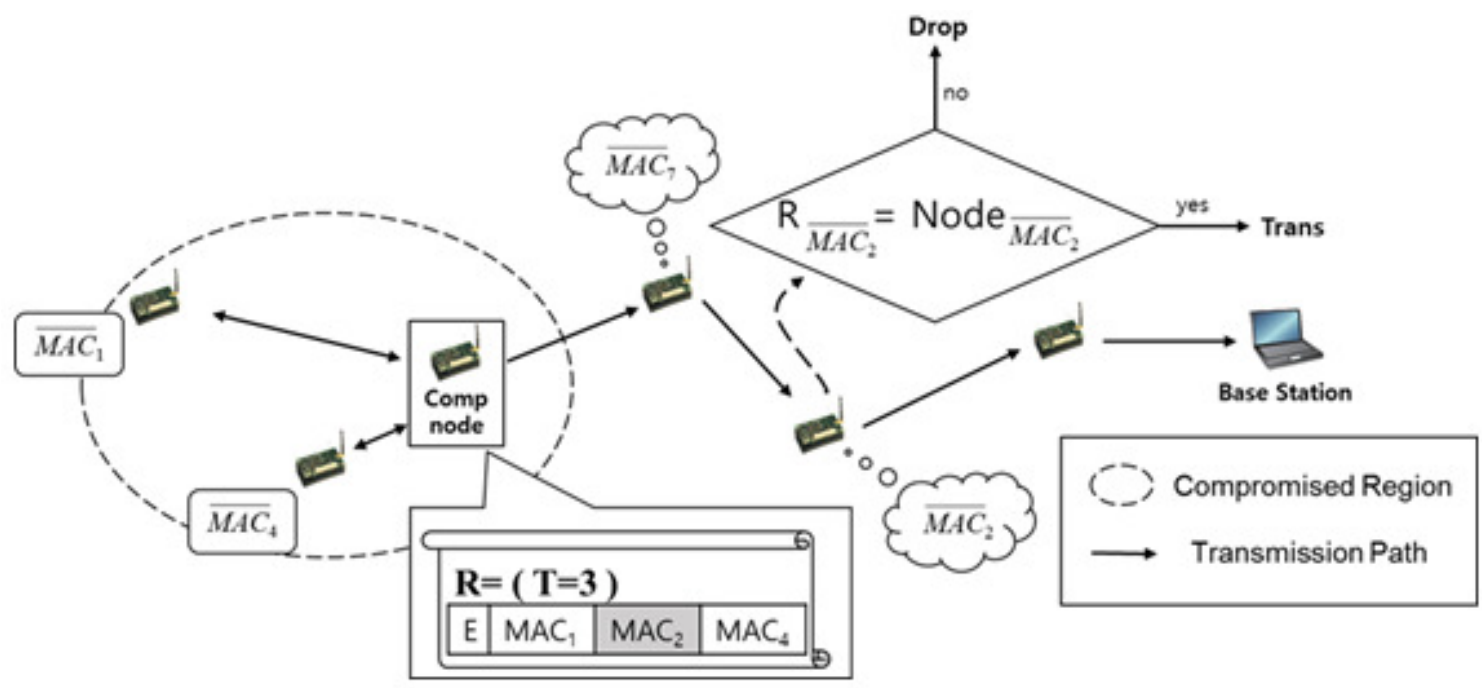

Figure 4. False report filtering process 
Figure 4 shows the false report filtering process. In Figure $4, \mathrm{MAC}_{\mathrm{n}}$ refers to the MAC belonging to $\mathrm{n}$ partitions. The attacker must compromise the same number of nodes as the threshold value to generate a complete false report using the compromised node. In Figure 4, assuming that the partition compromised two different nodes in a situation where the threshold value is $3, \mathrm{MAC}_{1}$ and $\mathrm{MAC}_{4}$ are known, but $\mathrm{MAC}_{2}$ is unknown. In the forwarding node, the node having $\mathrm{MAC}_{2}$ compares it to the verification report to verify the compromised MAC and drops the report. This mechanism can reduce unnecessary energy consumption by performing intermediate filtering of false reports.

\subsection{Motivation}

Setting an erroneous threshold according to the environment in the SEF has an adverse effect on the energy consumption efficiency [9]. To perform this task, research was conducted to determine the threshold value using fuzzy logic [8]. However, we did not consider the cycle of updating the threshold value for efficient use of this scheme. If the update period is wide, the node information value must be requested to the node every time, which is inefficient in an environment where the attack rate does not change. Conversely, if the period is narrow, it is inefficient in environments where the attack rate changes frequently. Therefore, in order to manage energy efficiently, it is important to determine the threshold update period. In particular, a method of determining the threshold update period is needed to establish an adaptive fuzzy system that can reduce energy consumption while maintaining security. In addition, the network manager analyzes the update cycle to help the nodes efficiently manage their energy.

\section{PROPOSED SCHEME}

\subsection{Assumptions}

It is assumed that the plurality of sensor nodes is randomly placed in the destination field and arranged closely to each other. The route path is set during the pre-deployment phase and is assumed to use single-path routing. Each sensor node has a unique identification number. Each time the $\mathrm{CH}$ nodes transmit node information to the BS, the BS knows the specific information of the $\mathrm{CH}$ node. The WSN uses a cluster approach, which is advantageous because it considers performance and limited resources [10]. One cluster consists of a cluster head node and nine member nodes. Each member node collects the event and notifies the cluster head node, and the cluster head sends the report to the BS.

\subsection{Overview}

We analyzed the power consumption depending on the update period to the experimental environment based on SEF. Threshold determination fuzzy logic uses the information transmitted at a particular node to determine the threshold. Fuzzy inference uses the min-max composition [11] of the mandani model, which is one of the inference models, and the center of area (COA) method is used for the defuzzification method. Figure 5 shows that the $\mathrm{CH}$ node receives specific data and determines a new threshold value for the environment according to the cycle from the evaluation function. The FTR(False Traffic Ratio), BS_H(Base Station Hop), and RE(Remaining Energy) were used as inputs. 


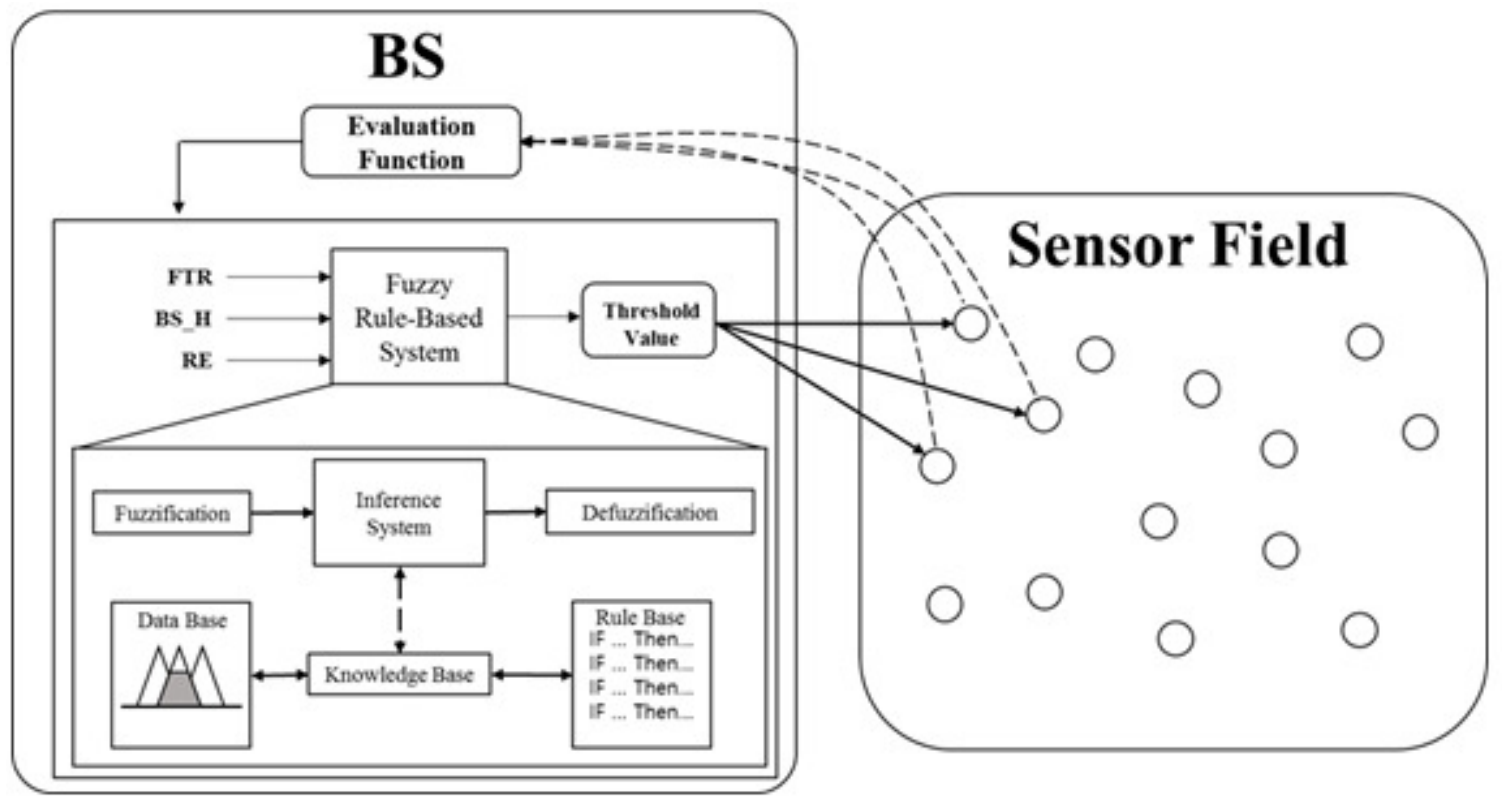

Figure 5. Overview of the proposed scheme

\subsection{Security Period Update Method}

In the initial period in the $\mathrm{CH}$, the $\mathrm{CH}$ transmits specific information such as the current set period value, the power consumption value of the node, and the attack rate to the BS. The BS transmits a new threshold value and period to the $\mathrm{CH}$ node after determining whether to update the update period through the received information and the evaluation function.

$$
f(x)=\left(\sqrt{(x-50)^{2}}\right)^{F}+\mathrm{MP}
$$

Equation 1. Evaluation Function

Equation (1) describes the evaluation function used in the proposed method, where $x$ is the measured attack rate, $F$ is the flexibility for period determination, and MP is the minimum period value. The BS evaluates the security rate for determining the new period. When the cycle is determined, the new threshold value is output using the fuzzy algorithm as shown below.

- False Traffic Ratio(FTR $)=\{$ L(Low $)$, M(Middle $), \mathbf{H}($ High $)\}$

- Base Station Hop $($ BS_H $)=\{$ VN(Very_Near $)$, N(Near $),$ M(Middle $),$ F(Far $)$, VF(Very_Far)\}

- Remaining Energy(RE) $=\{$ VL(Very_Low $)$, L(Low), H(High), VH(Very_High $)\}$

Figure 6 (a), (b), and (c) show the membership function to find the optimal threshold. The input values are defined as follows. 


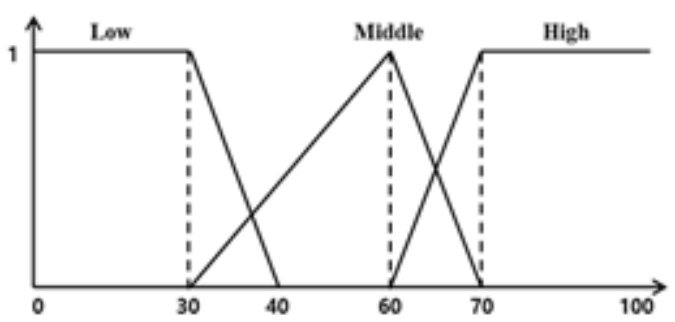

(a) False Traffic Ratio

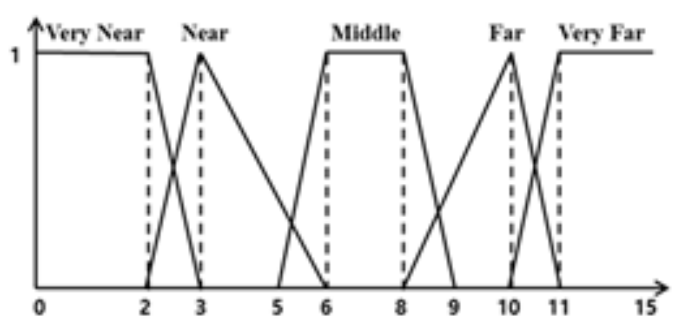

(b) BS Hop

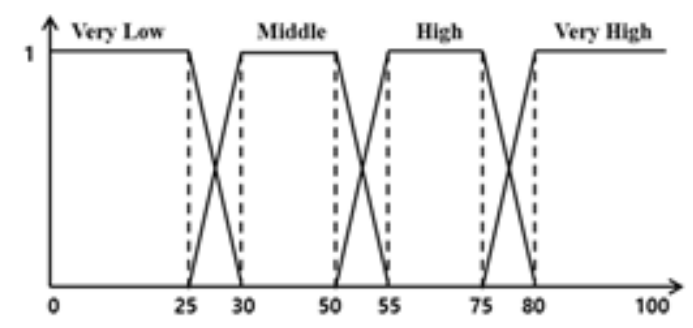

(c) Remaining Energy

Figure 6. Fuzzy membership functions

- (a) FTR: The attack rate measured per cycle, which is used to improve the filtering performance.

- (B) BS_H: The number of hops from the cluster head node to the BS. This can reduce energy consumption by considering the number of hops.

- (C) RE: This value is calculated as a percentage of the energy remaining in the node. It is used to save energy consumption and increase the node life time.

The proposed scheme adjusts the security strength and the energy consumption of the node by setting the attack rate, the energy state measured by the period, and the distance to the BS as the input value of the fuzzy system to set the new threshold value suitable for the current network situation. The new threshold and the new period are broadcast to the sensor network, as shown in Figure 5.

Table 1. Fuzzy if-then rules

\begin{tabular}{|c|c|c|c|c|}
\hline \multirow{2}{*}{ rule } & \multicolumn{3}{|c|}{ Input } & output \\
\cline { 2 - 5 } No. & FTR & $B S_{-} H$ & $R E$ & $T$ \\
\hline 7 & $L$ & $N$ & $H$ & $T 3$ \\
\hline 17 & $L$ & $V F$ & $V L$ & $T 2$ \\
\hline
\end{tabular}


Computer Science \& Information Technology (CS \& IT)

\begin{tabular}{|c|c|c|c|c|}
\hline 24 & $M$ & $V N$ & $V H$ & $T 2$ \\
\hline 41 & $M$ & $V F$ & $V H$ & $T 4$ \\
\hline 56 & $H$ & $F$ & $V H$ & $T 5$ \\
\hline
\end{tabular}

\section{EXPERIMENTAL RESULTS}

Table 2. Simulation parameters

\begin{tabular}{|c|c|c|}
\hline \multicolumn{2}{|l|}{ Parameters } & \multirow{2}{*}{$\begin{array}{l}\text { Value } \\
1,000 \mathrm{~m} \times 1,000 \mathrm{~m}\end{array}$} \\
\hline \multirow{5}{*}{ Network Environment } & Field Size & \\
\hline & Number of Nodes & 1,000 \\
\hline & Cluster Head Nodes & 100 \\
\hline & $\begin{array}{l}\text { Number of Events } \\
\text { (Discrete Occur) }\end{array}$ & 1,000 \\
\hline & Node Transmit Range & $100-150 \mathrm{~m}$ \\
\hline \multirow{3}{*}{ Transmit Size } & Report Size & $25+$ MAC Size \\
\hline & MAC Size & 1 byte \\
\hline & CH Node Info Size & 2 byte \\
\hline \multirow{5}{*}{ Energy Consumption } & Transmit & $16.25 \mu \mathrm{J}$ (per 1byte) \\
\hline & Receive & $12.5 \mu \mathrm{J}$ (per 1byte) \\
\hline & Report Generation & $70 \mu \mathrm{J}$ \\
\hline & MAC Generation & $15 \mu \mathrm{J}$ \\
\hline & Verification & $75 \mu \mathrm{J}$ \\
\hline \multirow{3}{*}{ Security Value } & SEF Threshold Value & $2-6$ \\
\hline & Key Number Per Node & 1 \\
\hline & Global Key Pool Size & 50 \\
\hline
\end{tabular}


In this section, we compared the performance of the SEF with the fuzzy update period through experiments. Table 2 shows the parameter values for the experiment. The node information was created based on the Micaz model [12]. The report size depends on the threshold value. The reason why the threshold value starts from 2 is that if the threshold value is 1 , even if only one node is damaged, a complete false report can be made. If the false report threshold is 1 , the BS cannot filter the false report. The threshold is updated every cycle and is determined by the fuzzy rule. The global key pool size is 50 and 5 partitions are used. Events occur 1,000 times at random locations.

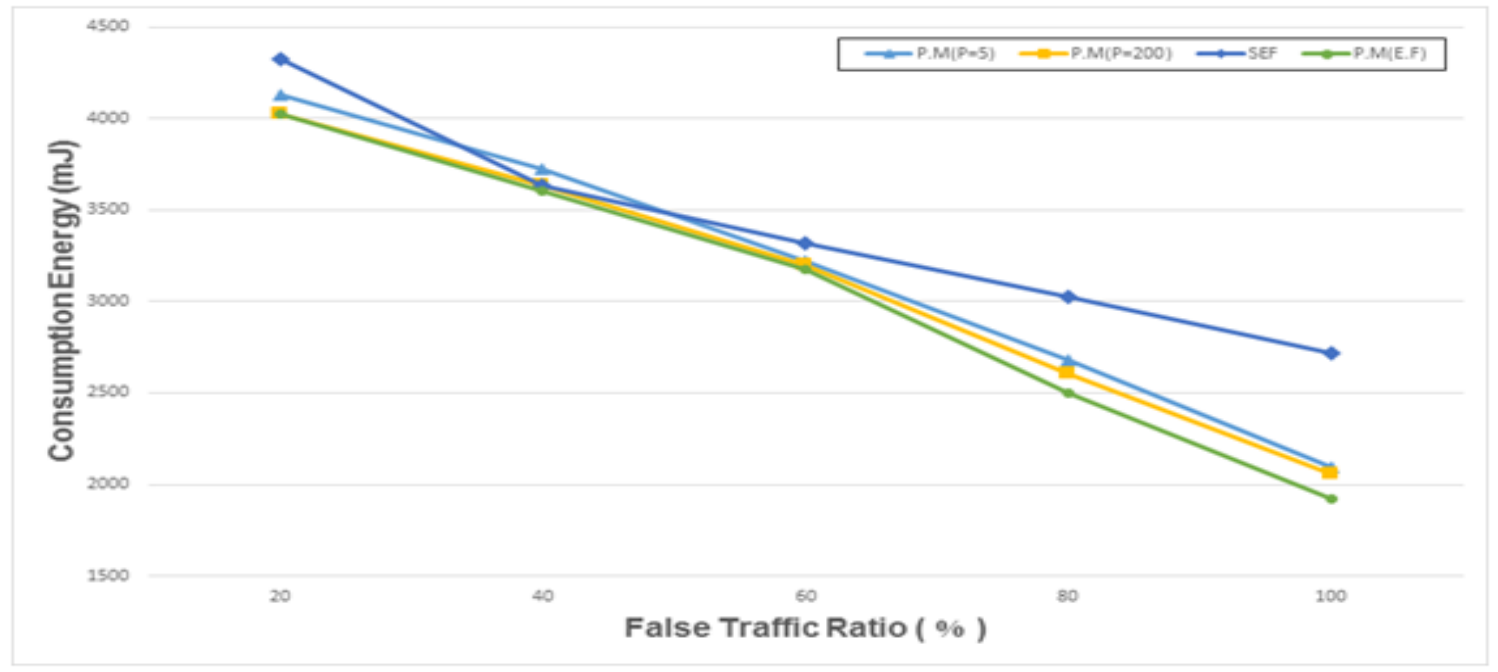

Figure 7. Energy consumption as a function of the FTR

Figure 7 shows the energy consumption of the FTR and fuzzy logic according to the threshold update period. If $\mathrm{P}$ is 200 , it means that the threshold value is adjusted every 200 events.

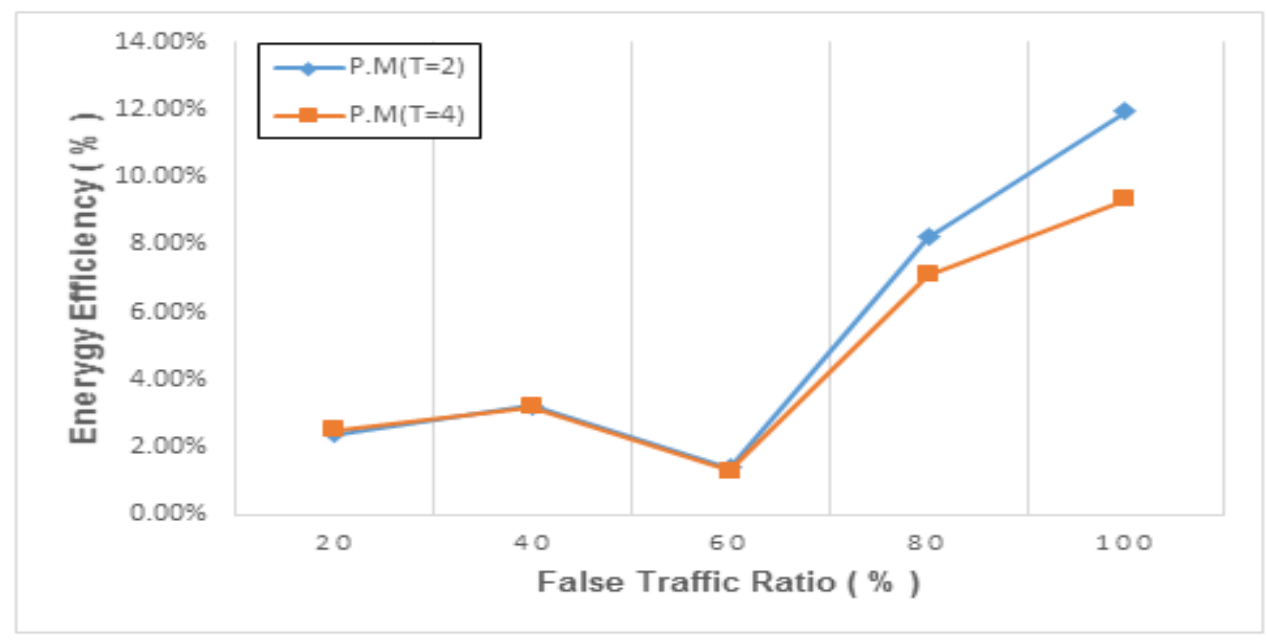

Figure 8. Increased energy efficiency with optimal cycles 
Figure 8 shows the graph of the energy efficiency comparison with the optimum cycles. When the thresholds are 2 and 4, the energy efficiency improved by up to $11.96 \%$ and $9.36 \%$, respectively. If the user applies the proposed technique in an area where the attack is high, it helps to save energy.

\section{CONCLUSIONS}

WSN is vulnerable to false report injection attacks because nodes are exposed to the open environment. To solve this problem, Fan Ye et al. proposed a SEF scheme that performs en-route filtering using a key. In statistical filtering techniques, thresholds affect energy management. Although research has been conducted to establish thresholds appropriate to the environment, energy management is adversely affected if the cycle is set incorrectly because the threshold update period is not taken into consideration. In this paper, we proposed a method of updating the threshold value through the evaluation function to update the appropriate period of the threshold value suitable for the network environment. The experimental results show that the energy efficiency increased by $11.96 \%$ with the optimum cycles.

\section{ACKNOWLEDGEMENTS}

This research was supported by Basic Science Research Program through the National Research Foundation of Korea (NRF) funded by the Ministry of Education, Science and Technology (No. NRF-2015R1D1A1A01059484).

\section{REFERENCES}

[1] Reejamol, K. J., and P. Dhanya Raj. "Hole handling techniques in wireless sensor networks: A survey." Computational Intelligence and Computing Research (ICCIC), 2016 IEEE International Conference on. IEEE, (2016)

[2] Nam, Su Man, and Tae Ho Cho. "A fuzzy rule-based path configuration method for LEAP in sensor networks." Ad Hoc Networks 31 (2015)

[3] Sen, Soumita, Chandreyee Chowdhury, and Sarmistha Neogy. "Design of cluster-chain based WSN for energy efficiency." Applied and Theoretical Computing and Communication Technology (iCATccT), 2016 2nd International Conference on. IEEE, (2016)

[4] Winkler, Thomas, and Bernhard Rinner. "Security and privacy protection in visual sensor networks: A survey." ACM Computing Surveys (CSUR) 47.1 (2014)

[5] Gupta, Pallavi, Vinay Prakash, and Preetam Suman. "Noticeable key points and issues of sensor deployment for large area Wireless Sensor Network: A survey." System Modeling \& Advancement in Research Trends (SMART), International Conference. IEEE, (2016)

[6] Alanwar, Amr, et al. "PrOLoc: resilient localization with private observers using partial homomorphic encryption: demo abstract." Proceedings of the 16th ACM/IEEE International Conference on Information Processing in Sensor Networks. ACM, (2017)

[7] F. Ye, H. Luo, S. Lu and L. Zhang, "Statistical en-route filtering of injected false data in sensor networks," Selected Areas in Communications, IEEE Journal on, vol. 23, pp. 839-850, (2005) 
[8] Kim, Cho. "Determination Method of Security Threshold using Fuzzy Logic for Statistical Filtering based Sensor Networks." Journal of the Korea Society for Simulation 16.2 27-35 (2007)

[9] Ahn, Cho. "A Correlation Analysis of the MAC Length in Statistical En-route Filtering based WSNS." International Journal of Advanced Research(IJAR) 4.8 (2016).

[10] Sahul, Ashwani, Bindiya, and Gursewak "Location Based-Balanced Clustering Algorithm for Wireless Sensor Network." International conference on Signal Processing, Communication, Power and Embedded System (SCOPES) (2016)

[11] Babuška, Robert. "Fuzzy systems, modeling and identification." Delft University of Technology, Department of Electrical Engineering Control Laboratory, Mekelweg 4 (1996).

[12] Quwaider, Muhannad. "Real-time intruder surveillance using low-cost remote wireless sensors." Information and Communication Systems (ICICS), 2017 8th International Conference on. IEEE, (2017)

\section{AUTHORS}

Jung Sub Ahn received a B.S. degree in Computer Engineering from Kyunil University in 2016 and is currently pursuing an M.S. degree in the Department of Electrical and Computer Engineering from Sungkyunkwan University.

Tea Ho Cho received a Ph.D. degree in Electrical and Computer Engineering from the University of Arizona, USA, in 1993, and B.S. and M.S. degrees in Electrical and Computer Engineering from Sungkyunkwan University, Republic of Korea, and the University of Alabama, USA, respectively. He is currently a professor at the College of Information and Communication Engineering, Sungkyunkwan University, Korea.
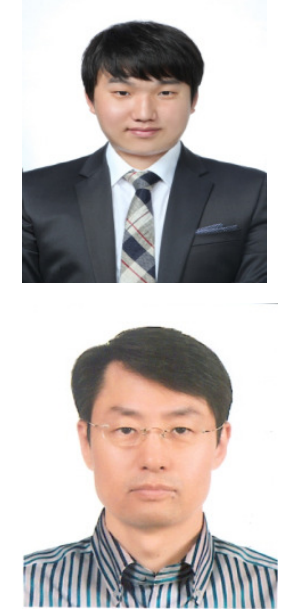